\title{
Diversification in Cotton Based Cropping System under Mechanization in Rainfed Condition of Vidarbha of Maharashtra, India
}

\author{
A.B. Turkhede*, M.B. Nagdeve, A.P. Karunakar, V.V. Gabhane, \\ V.D. Mohod and R.S. Mali \\ All India Coordinator Research Project for Dryland Agriculture, Dr. Panjabrao Deshmukh Krishi \\ Vidyapeeth, Akola (Maharashtra), India \\ *Corresponding author
}

\section{A B S T R A C T}

Keywords

Diversification, Sequence cropping, Sustainable yield and value index, System productivity and profitability.

Article Info

Accepted:

23 August 2017

Available Online:

10 September 2017
An experiment was conducted during 2013-14 to 2016-17 at All India Coordinator Research Project for Dryland Agriculture, Dr. Panjabrao Deshmukh Krishi Vidyapeeth, Akola (M.S.). Treatments consist of seven cropping patterns and two nutrients management tested in split plot design with three replications. On the basis of pooled analysis, the results revealed that, among the various treatments combination Cotton + soybean (4:10)safflower with application of $100 \%$ RDF (through inorganic fertilizer) recorded significantly higher cotton equivalent yield (2103 and $1824 \mathrm{~kg} \mathrm{ha}^{-}$ ${ }^{1}$ ), net monetary returns (Rs45850 and $33394 \mathrm{ha}^{-1}$ ), B:C ratio (2.01 and 1.75), RWUE (2.91 and 2.52), sustainable value and yield index (0.94 and 0.81 and 0.85 and 0.61 ), system productivity and profitability (8.25 and $7.43 \mathrm{~kg} \mathrm{ha}^{-1}$ day $^{-1}$ and 179.80 and $136.28 \mathrm{Rs} \mathrm{ha}^{-1}$ day $^{-1}$ ) than rest of the treatments combinations.

\section{Introduction}

In most multiple cropping systems developed by smallholders, productivity in terms of harvestable products per unit area is higher than under sole cropping with the same level of management. Yield advantages can range from 20 to $60 \%$ and accrue due to reduction of pest incidence and more efficient use of nutrients, water and solar radiation. Enhanced yields in diverse cropping systems may result from a variety of mechanisms such as more efficient use of resources (light, water, nutrients) or reduced pest damage.
Intercropping, which breaks down the monoculture structure, can provide pest control benefits, weed control advantages, reduced wind erosion, and improved water infiltration.

Indian agriculture is now facing second generation problems like rising or lowering of water table, nutrient imbalance, soil degradation, salinity, resurgence of pests and diseases, environmental pollution and decline in farm profit. Crop diversification shows lot 
of promise in alleviating these problems through fulfilling the basic needs and regulating farm income, withstanding weather aberrations, controlling price fluctuation, ensuring balanced food supply, conserving natural resources, reducing the chemical fertilizer and pesticide loads, environmental safety and creating employment opportunity. Diversification is gradually taking place as a consequence of either launching macroeconomic reforms in agriculture sector or rising domestic demand due to urbanization and increasing income levels. Crop diversification has been recognized as an effective strategy for achieving the objectives of food security, nutrition security, income growth, poverty alleviation and employment generation, judicious use of land and water resources, sustainable agricultural development and environmental improvement. The ability of the country to diversify the cropping pattern for attaining various goals depends on the opportunities available for diversification, the need for diversification and responsiveness of the farmers to these needs and opportunities. The opportunities for crop diversification emerge from technological breakthroughs, changes in demand pattern, development of irrigation, availability of marketing infrastructure and new trade arrangements.

Crop diversification through intercropping has been shown to improve crop productivity and profitability, conservation of resources and provide a kind of biological insurance against risks and aberrant rainfall behavior in rainfed condition (Dutta and Bandyopadhyay, 2006). Strip cropping is a form of intercropping comprises growing of soil conservation and soil depleting crops in alternate strips. Besides increasing overall productivity and income, intercropping of legumes with cereals/ millets/oilseeds/pulses helps in improving physical properties of soil and building up of soil fertility.
Diversification is the process to take advantage of emerging opportunities created by technology, new markets, changes in policy etc. to meet certain goals, challenges and threats and to reduce risk (Chand and Chauhan, 2002). Crop diversification is one of the major components of diversification in agriculture. Crop diversification may be adopted as a strategy for profit maximization through reaping the gains of complementary and supplementary relationships or in equating substitution and price ratios for competitive products. It also acts as a powerful tool in minimization of risk in farming. These considerations make a strong case for farm/crop diversification in India. (Gupta and Tewari, 1985).Crop diversification in India is generally viewed as a shift from traditionally grown less remunerative crops to more remunerative crops.

Market infrastructure development and certain other price related supports also induce crop shift. Higher profitability and also the resilience/stability in production also induce crop diversification. Crop diversification and large number of crops are practiced in rainfed areas to reduce the risk factor of crop failures due to drought. Crop substitution and shift are also taking place in the areas with distinct soil problems for example, the growing of rice in high water table areas replacing pulses and cotton, promotion of soybean in place of sorghum in Vertisols (medium and deep black soils).

Thus, the present investigation was undertaken to "Diversification in cotton based cropping system under mechanization in dry land condition with the objectives of to intensify and diversify the system to meet out domestic needs of small farmers with find out the most suitable rabi crop for cotton-soybean based sequence cropping and to evaluate the economics of different cropping system. 


\section{Materials and Methods}

The field study was carried out during the kharif season of 2013-14 to 2016-17 at All India Coordinator Research Project for Dryland Agriculture, Dr. Panjabrao Deshmukh Krishi Vidyapeeth, Akola (M.S.) on a clayey soil with FC 34.2 (\%), PWP 14.2 (\%), BD1.36 $\left(\mathrm{Mg} \mathrm{M}^{-3}\right)$ and depth of soil was $132 \mathrm{~cm}$. Soil was medium in available nitrogen $\left(182 \mathrm{~kg} \mathrm{ha}^{-1}\right)$ and phosphorus(19.8 $\mathrm{kg} \mathrm{ha} \mathrm{a}^{-1}$ ) and high in available potassium (341.6 $\mathrm{kg} \mathrm{ha}^{-1}$ ) with $\mathrm{pH} \mathrm{7.8,} \mathrm{EC} 0.2$ and organic carbon of soil $4.7 \mathrm{~g} \mathrm{~kg}^{-1}$. Experiment consists of seven cropping patterns treatments in main plot $\mathrm{C}_{1^{-}}$Sole cotton, $\mathrm{C}_{2}$-Sole soybean-chickpea, $\mathrm{C}_{3}$ - Sole soybean-linseed, $\mathrm{C}_{4}$-Solesoybean-safflower, $\mathrm{C}_{5}$-Cotton + soybean (4:10)-chickpea, $\mathrm{C}_{6}$-Cotton + soybean (4:10)-linseed and $\mathrm{C}_{7}$-Cotton + soybean (4:10)-safflower and two nutrients management treatments in sub plot i.e. $\mathrm{F}_{1^{-}} 100$ $\% \mathrm{RDF}$ and $\mathrm{F}_{2}-50 \% \mathrm{~N}+100 \% \mathrm{P}$ and $\mathrm{K}$ applied through inorganic fertilizer $+50 \% \mathrm{~N}$ through gliricidia leaves loping at one month after sowing.

After the harvest of soybean crop, land was prepared with the help of tractor drawn rotatvator and rabi season crops are also sown with tractor drawn seed drill. An application of 100 per cent RDF applied to the treatment of

cotton/soybean/chickpea/linseed/safflower and 55 per cent RDF (on area basis) applied to the treatment of cotton + soybean (4:10)chickpea /linseed/safflower crops. In kharif season, 45:55:30 $\mathrm{kg} \mathrm{ha}^{-1}$ NPK was applied to the treatment of cotton + soybean $(4: 10)$ row proportions.

Total rainfall received during the cropping period of experiments was 860.7, 512.2, 518.7 and 724.1 in $45,28,26$ and 43 rainy days in 2013-14, 2014-15, 2015-16 and 2016-17 respectively. During the year 2014-15, trial was vitiated due to late onset of monsoon i.e. 16-22 July (29 MW) and due to late harvested of soybean crop rabi crops were not possible to sown in proper sowing time. Therefore, in pooled analysis the yield of $2^{\text {nd }}$ year was not considered.

\section{Results and Discussion}

\section{Yield}

On the basis of three years data, results showed that, seed cotton yield, grain yield of soybean, chickpea, linseed and safflower was recorded higher in sole cropping system than yield obtained in the treatment of cotton + soybean (4:10) row proportion (i.e. 1550, $1825,668,119$ and $1078 \mathrm{~kg} \mathrm{ha}^{-1}$, respectively (Tables 1, 2 and 3).

An application of 100\% RDF recorded higher seed cotton and soybean yield (1129 and $\left.1479 \mathrm{~kg} \mathrm{ha}^{-1}\right)$. The results showed that yield of soybean, chickpea, linseed and safflower were more or less equal in grain as well as in fodder yield. Reduction in yield of cotton /soybean/chickpea/linseed/safflower crops in treatment of cotton + soybean (4:10) row proportion can be attributed to the less number of plants per unit area. Similar, trend of results also recorded in the stalk yield of cotton and straw yield of soybean and also in straw yield of rabi crops.

\section{Cotton equivalent yield}

During the year 2013-14, in respect of cotton equivalent yield, the treatments of cotton + soybean (4:10)-safflower, cotton + soybean (4:10) - chickpea and sole soybean- safflower were being at par (i.e. 2252, 2231 and $2223 \mathrm{~kg}$ $\mathrm{ha}^{-1}$, respectively) and significantly superior than the rest of the treatments. During the year 2015-16, the treatments of cotton + soybean (4:10)-safflower and cotton + soybean (4:10)- chickpea were being at par 
and significantly superior than the rest of the treatments i.e. 1677 and $1653 \mathrm{~kg} \mathrm{ha}^{-1}$ respectively. During the year 2016-17, the treatments of cotton + soybean (4:10) safflower was recorded significantly higher cotton equivalent yield and found significantly superior over rest of the treatments i.e. $2192 \mathrm{~kg} \mathrm{ha}^{-1}$. However, in pooled analysis, results showed that, the treatments of cotton + soybean (4:10) safflower and sole soybean - safflower was being at par with each other and significantly superior than rest of treatments i.e. 2103 and $2041 \mathrm{~kg} \mathrm{ha}^{-1}$ (Table 4).

This might be due to favorable effect of soybean and succeeding rabi crops on cotton growth as there was no intercrop competition for growth due to the strip sowing. When cotton sown with soybean in kharif season and safflower in rabi season, this attributed to better productivity of cotton, soybean and safflower recorded the higher cotton equivalent yield due to the higher remunerative and better market prices. Increase productivity of cotton with additional yield of mixed crops of soybean and rabi crops specially the yield of safflower helped in increasing the cotton equivalent yield over treatments of sole cotton. Maize + soybean French bean cropping system gave higher equivalent yield compared to other cropping systems. These results are in conformity with the findings of Chittapur (2004), Gill and Ahlawat, (2006) and Sankaranarayanan et al., (2012).

An application of $100 \%$ RDF recorded significantly higher cotton equivalent yield during the year 2013-14, 2015-16 and in pooled analysis i.e. 2027, 1505 and $1824 \mathrm{~kg}$ $\mathrm{ha}^{-1}$, respectively. However, during the years of 2016-17, seed cotton equivalent yield was found to be non-significant. Interaction effect was found to be non-significant. This was due to inorganic fertilizer application to cotton and its residual effect on safflower. The yield increase was attributed to significant increase in plant height, sympodial branches plant ${ }^{-1}$, bolls plant ${ }^{-1}$ and seed cotton yield boll ${ }^{-1}$ with recommended dose of fertilizer. These results are in close agreement with the findings of Gawai and Pawar (2005). Similar results are also reported by Tomar and Julka (1997) and Tomar et al., (2000).

\section{Economic}

During the year 2013-14, in respect of gross monetary returns, the results revealed that the treatments of cotton + soybean (4:10)chickpea, cotton + soybean (4:10)-safflower and soybean- safflower were being at par recorded significantly superior than rest of the treatments (Rs. 121100, 120853 and 116982 $\left.\mathrm{ha}^{-1}\right)$. During the years 2015-16, gross monetary returns was recorded significantly higher value with the treatments of cotton + soybean (4:10) - safflower and found being at par with the treatments of cotton+ soybean (4:10) - chickpea (Rs. 82661 and 81567 ha $^{-1}$ ) than rest of the treatments. During the year 2016-17, the treatments of cotton + soybean (4:10) - safflower was significantly superior to the rest of the treatments i.e. Rs $94825 \mathrm{ha}^{-1}$. However, in pooled analysis the treatments of cotton + soybean (4:10)-safflower and sole soybean-safflower found being at par with each other's and recorded significantly higher gross monetary returns compared to the rest of the treatments i.e. Rs. 91147 and 87887 ha $^{-1}$, respectively.

During the years 2013-14 and 2015-16, the economics analysis of the system revealed that highest monetary advantage in terms of net monetary returns was recorded significantly higher value with the treatments of cotton + soybean (4:10)-safflower and cotton + soybean $(4: 10)$-chickpea which was found being at par with each other and significantly higher than rest of the treatments 
i.e. Rs. 84574 and 81267and Rs.41752 and $37587 \mathrm{ha}^{-1}$. However, during the years 201617 and also in pooled analysis, cotton + soybean (4:10)-safflower recorded significantly higher net monetary returns than rest of the treatments (Rs. 48929 and 45850 $\mathrm{ha}^{-1}$ ).

In respect of nutrient management treatments, during the year 2013-14, 2015-16 and in pooled analysis, $100 \%$ RDF recorded significantly higher gross monetary returns than $50 \% \mathrm{~N}+100 \% \mathrm{P}$ and $\mathrm{K}$ applied through inorganic fertilizer $+50 \% \mathrm{~N}$ through gliricidia. However, result was found to be non-significant during the year 2016-17. Interaction effect was found to be non significant (Table 5).

In respect of nutrient management treatments, during the year 2013-14, 2015-16, 2016-17 and in pooled analysis, treatment of $100 \%$ RDF recorded significantly higher net monetary returns than $50 \% \mathrm{~N}+100 \% \mathrm{P}$ and $\mathrm{K}$ applied through inorganic fertilizer $+50 \%$ $\mathrm{N}$ through gliricidia i.e. Rs. 73009, 31954, 32702 and $33394 \mathrm{ha}^{-1}$,respectively.

During the years 2013-14 to 2016-17 and also in pooled analysis, the economics of the system revealed that highest $\mathrm{B}$ : $\mathrm{C}$ ratio value recorded with the treatment of cotton + soybean (4:10)-safflower i.e. 3.33, 2.12, 2.07 and 2.01, respectively (Table 7).

In respect of nutrient management treatments from the year 2013-14 to 2016-17 and also in pooled analysis, $100 \%$ RDF recorded higher value of $\mathrm{B}: \mathrm{C}$ ratio i.e. 3.08, 1.82, 1.73 and 1.75 compared to the treatment of $50 \% \mathrm{~N}$ $+100 \% \mathrm{P}$ and $\mathrm{K}$ applied through inorganic fertilizer $+50 \% \mathrm{~N}$ through Gliricidia. Interaction effects found to be non-significant in respect of GMR, NMR and B: C ratio.

This might be due to favorable effect of soybean and succeeding rabi crops on cotton growth as there was no intercrop competition for growth due to the strip sowing. Due to more productivity and market rates of the mixed crops and low cost of cultivation also helped in increasing higher economics returns. Tanaka et al., (2007) reported that, crop sequence has a significant effect on cropping system net returns. A cropping systems approach may offer opportunities for producers to increase economic returns. Management of dynamic cropping systems will need to be based not only on single-year profit opportunities, but also on subsequent crop sequence effects. Maximum net returns was recorded at hirsutum cotton - mustard sequence followed by hirsutum cotton-wheat and arboretum cotton-wheat sequence than sole cotton cropping system also reported by Venugopal et al.,(2000). Cotton + sorghumragi, followed by cotton-sunflower -ragi and cotton-maize -ragi sequences were more profitable and economically viable than sole cotton (Jagvirsingh et al., 2000).

\section{Rain water use efficiency}

During the year 2013-14, results showed that, treatments of cotton+ soybean (4:10)safflower, Cotton + soybean (4:10)- chickpea, sole soybean - safflower and sole soybean chickpea cropping system were being at par and recorded significantly higher water use efficiency than rest of the treatments i.e.2.75, $2.73,2.72$ and $2.43 \mathrm{~kg} \mathrm{ha}^{-1} \mathrm{~mm}^{-1}$. During the year 2015-16, Cotton + soybean (4:10)safflower and Cotton + soybean (4:10)chickpea were being at par and recorded significantly higher RWUE than rest of the treatments i.e. 2.93 and $2.89 \mathrm{~kg} \mathrm{ha}^{-1} \mathrm{~mm}^{-1}$. During the year 2016-17, treatments of cotton + soybean (4:10)-safflower recorded significantly higher water use efficiency than rest of the treatments i.e. 3.03. However, in pooled analysis, the results showed that, cotton + soybean (4:10) - safflower and sole soybean - safflower cropping system were being at par $\left(2.91\right.$ and $\left.2.82 \mathrm{~kg} \mathrm{ha}^{-1} \mathrm{~mm}^{-1}\right)$ and 
recorded significantly higher water use efficiency than rest of the treatments. The rainwater use efficiency attended with cotton based sequence intercropping system, in general was higher as compare to the RWUE attained with sole crops. This indicated higher resource use efficiency of both rainfall and soil moisture by the component crops during the crop season. This might be due to higher grain yields of both the crops than the amount of water used for biomass production (Table 8).

Consumptive use and rate of moisture use were higher in the intercropping system than sole crop because both the crops absorbed more moisture during the crop period. Higher water use efficiency has been reported for maize-soybean and maize- mungbean (De and
Singh, 1981), maize-cowpea (Hulugalle and Lal, 1986), Maize + potato (Bharati et al., 2007), pearlmillet + greengram and pearlmillet + cowpea (Goswami et al., 2002) intercrops in relation to their respective monocrops. Tetarwal and Rana (2006) one row of mothbean in paired row of pearlmillet + and one row of greengram between paired rows of pigeonpea recorded higher water use efficiency over sole crop, respectively.

Water use efficiency recorded significantly higher in the treatment of $100 \%$ RDF than $50 \% \mathrm{~N}+100 \% \mathrm{P}$ and $\mathrm{K}$ applied through inorganic fertilizer $+50 \% \mathrm{~N}$ through Gliricidia during the year 2015-16 and in pooled analysis (2.63 and 2.52). Whereas, during the year 2013-14 and 2016-17, results did not showed the significant difference.

Table.1 Seed cotton yield and cotton stalk yield as influenced by cropping pattern and nutrient management

\begin{tabular}{|c|c|c|c|c|c|c|c|c|c|c|c|}
\hline \multicolumn{2}{|c|}{ Treatments } & \multicolumn{5}{|c|}{ Seed Cotton Yield $\left(\mathrm{kg} \mathrm{ha}^{-1}\right)$} & \multicolumn{5}{|c|}{ Cotton Stalk Yield $\left(\mathrm{kg} \mathrm{ha}^{-1}\right)$} \\
\hline & & $\begin{array}{c}2013- \\
14\end{array}$ & $\begin{array}{c}2014- \\
15\end{array}$ & $\begin{array}{c}2015- \\
16\end{array}$ & $\begin{array}{c}2016- \\
17\end{array}$ & Mean & $\begin{array}{c}2013- \\
14\end{array}$ & $\begin{array}{c}2014- \\
15\end{array}$ & $\begin{array}{c}2015- \\
16\end{array}$ & $\begin{array}{c}2016- \\
17\end{array}$ & Mean \\
\hline A. & Main Plot (Cro & ing Pat & & & & & & & & & \\
\hline $\mathrm{C}_{1}$ & Sole cotton & 1489 & 985 & 1331 & 1832 & 1550 & 3115 & 1152 & 2076 & 2418 & 2536 \\
\hline $\mathrm{C}_{2}$ & $\begin{array}{l}\text { Sole soybean- } \\
\text { chickpea }\end{array}$ & - & - & - & - & - & - & - & - & - & - \\
\hline $\mathrm{C}_{3}$ & $\begin{array}{l}\text { Sole soybean- } \\
\text { linseed }\end{array}$ & - & - & - & - & - & - & - & - & - & - \\
\hline $\mathrm{C}_{4}$ & $\begin{array}{l}\text { Sole soybean- } \\
\text { safflower }\end{array}$ & - & - & - & - & - & - & - & - & - & - \\
\hline $\mathrm{C}_{5}$ & $\begin{array}{l}\text { Cotton }+ \\
\text { soybean }(4: 10)- \\
\text { chickpea }\end{array}$ & 957 & 489 & 804 & 960 & 907 & 2061 & 716 & 1254 & 1267 & 1527 \\
\hline $\mathrm{C}_{6}$ & $\begin{array}{l}\text { Cotton }+ \\
\text { soybean }(4: 10)- \\
\text { Linseed }\end{array}$ & 954 & 485 & 800 & 951 & 902 & 1958 & 643 & 1249 & 1255 & 1487 \\
\hline $\mathrm{C}_{7}$ & $\begin{array}{l}\text { Cotton + } \\
\text { soybean(4:10)- } \\
\text { Safflower }\end{array}$ & 958 & 487 & 806 & 971 & 911 & 2008 & 607 & 1257 & 1281 & 1515 \\
\hline B. & Sub Plot -Nutri & t Mana & ment & & & & & & & & \\
\hline $\mathrm{F}_{1}$ & $100 \%$ RDF & 1176 & 655 & 992 & 1220 & 1129 & 2485 & 824 & 1547 & 1703 & 1881 \\
\hline$\overline{F_{2}}$ & $\begin{array}{l}50 \% \mathrm{~N}+100 \\
\% \mathrm{P} \text { and } \mathrm{K} \\
\text { applied } \\
\text { through } \\
\text { inorganic } \\
\text { fertilizer }+50 \\
\% \mathrm{~N} \text { through } \\
\text { Gliricidia }\end{array}$ & 1003 & 568 & 879 & 1137 & 1006 & 2086 & 736 & 1371 & 1729 & 1632 \\
\hline
\end{tabular}


Table.2 Soybean grain yield and soybean straw yield as influenced by cropping pattern and nutrient management

\begin{tabular}{|c|c|c|c|c|c|c|c|c|c|c|c|}
\hline \multirow{2}{*}{\multicolumn{2}{|c|}{ Treatments }} & \multirow{2}{*}{\multicolumn{5}{|c|}{ Soybean Grain Yield $\left(\mathrm{Kg} \mathrm{ha}^{-1}\right)$}} & \multirow{2}{*}{\multicolumn{5}{|c|}{ Soybean Straw Yield(kg/ha) }} \\
\hline & & & & & & & & & & & \\
\hline A. & \multicolumn{4}{|c|}{ Main Plot (Cropping Pattern) } & \multirow{2}{*}{$\begin{array}{c}2016-17 \\
- \\
\end{array}$} & \multirow{2}{*}{$\begin{array}{c}\text { Mean } \\
- \\
\end{array}$} & \multirow{2}{*}{$\begin{array}{c}2013-14 \\
- \\
\end{array}$} & \multirow{2}{*}{$\begin{array}{c}\text { 2014-15 } \\
- \\
-\end{array}$} & \multirow{2}{*}{$\begin{array}{r}2015-16 \\
- \\
\end{array}$} & \multirow{2}{*}{$\begin{array}{c}2016-17 \\
- \\
\end{array}$} & \multirow{2}{*}{$\begin{array}{c}\text { Mean } \\
- \\
\end{array}$} \\
\hline $\mathrm{C}_{1}$ & Sole cotton & - & - & - & & & & & & & \\
\hline $\mathrm{C}_{2}$ & $\begin{array}{ll}\begin{array}{l}\text { Sole } \\
\text { chickpea }\end{array} & \text { soybean- } \\
\end{array}$ & 2459 & 945 & 1075 & 1943 & 1825 & 1819 & 1129 & 1311 & 2160 & 1763 \\
\hline $\mathrm{C}_{3}$ & $\begin{array}{ll}\text { Sole } & \text { soybean- } \\
\text { linseed } & \end{array}$ & 2459 & 945 & 1075 & 1943 & 1825 & 1819 & 1129 & 1311 & 2160 & 1763 \\
\hline $\mathrm{C}_{4}$ & $\begin{array}{l}\text { Sole soybean- } \\
\text { safflower }\end{array}$ & 2459 & 945 & 1075 & 1943 & 1825 & 1819 & 1129 & 1311 & 2160 & 1763. \\
\hline $\mathrm{C}_{5}$ & $\begin{array}{ll}\text { Cotton } & + \\
\text { soybean(4:10)- } \\
\text { chickpea }\end{array}$ & 1484 & 611 & 613 & 1155 & 1084 & 1215 & 939 & 747 & 1284 & 1082 \\
\hline $\mathrm{C}_{6}$ & $\begin{array}{l}\text { Cotton } \\
\text { soybean(4:10)- } \\
\text { Linseed }\end{array}$ & 1509 & 641 & 616 & 1115 & 1080 & 1216 & 938 & 752 & 1240 & 1069 \\
\hline $\mathrm{C}_{7}$ & $\begin{array}{ll}\text { Cotton } & + \\
\text { soybean(4:10)- } & \\
\text { Safflower } & \end{array}$ & 1500 & 634 & 619 & 1161 & 1093 & 1294 & 935 & 755 & 1291 & 1113 \\
\hline B. & \multicolumn{11}{|c|}{ Sub Plot -Nutrient Management } \\
\hline $\mathrm{F}_{1}$ & $100 \%$ RDF & 2025 & 811 & 879 & 1532 & 1479 & 1559 & 1063 & 1073 & 1703 & 1448 \\
\hline $\mathrm{F}_{2}$ & $\begin{array}{l}50 \% \mathrm{~N}+100 \% \mathrm{P} \\
\text { and } \mathrm{K} \text { applied } \\
\text { through inorganic } \\
\text { fertilizer }+50 \% \mathrm{~N} \\
\text { through Gliricidia }\end{array}$ & 1931 & 763 & 812 & 1554 & 1432 & 1491 & 1004 & 990 & 1729 & 1403 \\
\hline
\end{tabular}

Table.3 Chickpea, linseed and safflower grain yield and straw yield as influenced by cropping pattern and nutrient management

\begin{tabular}{|c|c|c|c|c|c|c|c|c|c|c|c|}
\hline \multicolumn{2}{|c|}{ Treatments } & 13-14 & 14-15 & 15-16 & 16-17 & Mean & 13-14 & 14-15 & $15-16$ & 16-17 & Mean \\
\hline A. & Cropping Pattern & \multicolumn{5}{|c|}{ Chickpea Yield(Kg ha $\left.{ }^{-1}\right)$} & \multicolumn{5}{|c|}{ Chickpea Straw Yield $\left(\mathrm{Kg} \mathrm{ha}^{-1}\right)$} \\
\hline $\mathrm{C}_{2}$ & Sole soybean-chickpea & 1023 & - & 1018 & 344 & 668 & 825 & - & 793 & 385 & 574 \\
\hline $\mathrm{C}_{5}$ & $\begin{array}{lll}\text { Cotton } & \text { +sobean } & (4: 10)- \\
\text { Chickpea }\end{array}$ & 563 & - & 540 & 201 & 412 & 485 & - & 669 & 224 & 372 \\
\hline B. & Nutrient Management & & & & & & & & & & \\
\hline $\mathrm{F}_{1}$ & $100 \%$ RDF & 815 & - & 722 & 284 & 539 & 682 & - & 600 & 318 & 465 \\
\hline $\mathrm{F}_{2}$ & $\begin{array}{l}50 \% \mathrm{~N}+100 \% \mathrm{P} \text { and } \mathrm{K} \\
\text { applied through inorganic } \\
\text { fertilizer }+50 \% \mathrm{~N} \text { through } \\
\text { Gliricidia }\end{array}$ & 772 & - & 837 & 261 & 541 & 627 & - & 648 & 291 & 461 \\
\hline B. & Cropping Pattern & \multicolumn{5}{|c|}{ Linseed Yield $\left(\mathrm{Kg} \mathrm{ha}^{-1}\right)$} & \multicolumn{5}{|c|}{ Linseed Straw Yield $\left(\mathrm{Kg} \mathrm{ha}^{-1}\right)$} \\
\hline $\mathrm{C}_{3}$ & Sole soybean-Linseed & 175 & - & 170 & 71 & 119 & 188 & - & 197 & 79 & 167 \\
\hline $\mathrm{C}_{6}$ & $\begin{array}{l}\text { Cotton } \quad+\text { sobean } \quad(4: 10)- \\
\text { Linseed }\end{array}$ & 91 & - & 105 & 51 & 89 & 93 & - & 107 & 57 & 100 \\
\hline B. & Nutrient Management & & & & & & & & & & \\
\hline$F_{1}$ & $100 \%$ RDF & 131 & - & 129 & 61 & 120 & 135 & - & 153 & 68 & 135 \\
\hline $\mathrm{F}_{2}$ & $\begin{array}{l}50 \% \mathrm{~N}+100 \% \mathrm{P} \text { and } \mathrm{K} \\
\text { applied through inorganic } \\
\text { fertilizer }+50 \% \mathrm{~N} \text { through } \\
\text { Gliricidia }\end{array}$ & 134 & - & 146 & 61 & 118 & 146 & - & 150 & 68 & 132 \\
\hline C. & Cropping Pattern & \multicolumn{5}{|c|}{ Safflower Yield $\left(\mathrm{Kg} \mathrm{ha}^{-1}\right)$} & \multicolumn{5}{|c|}{ Safflower Straw Yield(Kg ha $\left.{ }^{-1}\right)$} \\
\hline $\mathrm{C}_{4}$ & Sole soybean- Safflwer & 1102 & - & 1117 & 965 & 1078 & 735 & - & 1101 & 815 & 968 \\
\hline $\mathrm{C}_{7}$ & $\begin{array}{l}\text { Cotton +sobean } \quad \text { (4:10)- } \\
\text { Safflower }\end{array}$ & 662 & - & 625 & 514 & 605 & 455 & - & 608 & 648 & 600 \\
\hline B. & Nutrient Management & & & & & & & & & & \\
\hline $\mathrm{F}_{1}$ & $100 \%$ RDF & 907 & - & 913 & 788 & 840 & 617 & - & 824 & 824 & 796 \\
\hline $\mathrm{F}_{2}$ & $\begin{array}{l}50 \% \mathrm{~N}+100 \% \mathrm{P} \text { and } \mathrm{K} \\
\text { applied through inorganic } \\
\text { fertilizer }+50 \% \mathrm{~N} \text { through } \\
\text { Gliricidia }\end{array}$ & 857 & - & 829 & 765 & 843 & 573 & - & 785 & 806 & 772 \\
\hline
\end{tabular}


Table.4 Cotton equivalent yield as influenced by cropping pattern and nutrient management

\begin{tabular}{|c|c|c|c|c|c|c|}
\hline & \multirow[t]{2}{*}{ Treatments } & \multicolumn{5}{|c|}{ Cotton equivalent yield $\left(\mathrm{kg} \mathrm{ha}^{-1}\right)$} \\
\hline & & 2013-14 & 2014-15 & 2015-16 & 2016-17 & Pooled \\
\hline A. & Main Plot (Cropping Pattern) & & & & & \\
\hline $\mathrm{C}_{1}$ & Sole cotton & 1489 & 985 & 1331 & 1832 & 1550 \\
\hline $\mathrm{C}_{2}$ & Sole soybean - Chickpea & 1989 & 795 & 1490 & 1552 & 1725 \\
\hline $\mathrm{C}_{3}$ & Sole soybean - Linseed & 1601 & 795 & 1075 & 1359 & 1361 \\
\hline $\mathrm{C}_{4}$ & Sole soybean - Safflower & 2223 & 795 & 1510 & 2032 & 2041 \\
\hline $\mathrm{C}_{5}$ & Cotton + soybean (4:10)- Chickpea & 2231 & 1003 & 1653 & 1880 & 1943 \\
\hline $\mathrm{C}_{6}$ & Cotton + soybean(4:10)-Linseed & 1956 & 1025 & 1410 & 1741 & 1708 \\
\hline $\mathrm{C}_{7}$ & Cotton + soybean $(4: 10)$-Safflower & 2252 & 1021 & 1677 & 2192 & 2103 \\
\hline & S.Em.土 & 44.0 & 12.6 & 45.2 & 39.8 & 27.9 \\
\hline & C.D.at $5 \%$ & 135.6 & 39.1 & 139.9 & 122.8 & 86.2 \\
\hline B. & Sub Plot -Nutrient Management & & & & & \\
\hline $\mathrm{F}_{1}$ & $100 \% \mathrm{RDF}$ & 2027 & 959 & 1505 & 1821 & 1824 \\
\hline $\mathrm{F}_{2^{-}}$ & $\begin{array}{l}50 \% \mathrm{~N}+100 \% \mathrm{P} \text { and } \mathrm{K} \text { applied through } \\
\text { inorganic fertilizer }+50 \% \mathrm{~N} \text { through Gliricidia }\end{array}$ & 1899 & 875 & 1394 & 1776 & 1728 \\
\hline & S.Em. \pm & 22.6 & 4.25 & 27.3 & 31.1 & 17.0 \\
\hline & C.D.at $5 \%$ & 68.8 & 13.09 & 82.8 & NS & 52.4 \\
\hline C. & Interaction effect & & & & & \\
\hline & S.Em. \pm & 60.0 & 29.7 & 72.3 & 82.1 & 45.1 \\
\hline & C.D.at $5 \%$ & $\mathrm{NS}$ & NS & $\mathrm{NS}$ & $\mathrm{NS}$ & NS \\
\hline
\end{tabular}

Table.5 Gross monetary returns as influenced by cropping pattern and nutrient management

\begin{tabular}{|c|c|c|c|c|c|c|}
\hline & \multirow{2}{*}{ Treatments } & \multicolumn{5}{|c|}{ Gross monetary returns $\left(\mathrm{Rs} \mathrm{ha}^{-1}\right)$} \\
\hline & & 2013-14 & 2014-15 & $2015-16$ & 2016-17 & Pooled \\
\hline A. & \multicolumn{6}{|l|}{ Main Plot (Cropping Pattern) } \\
\hline $\mathrm{C}_{1}$ & Sole cotton & 81768 & 38576 & 65155 & 79029 & 67515 \\
\hline $\mathrm{C}_{2}$ & Sole soybean - Chickpea & 105204 & 31636 & 74057 & 67611 & 74500 \\
\hline $\mathrm{C}_{3}$ & Sole soybean - Linseed & 84620 & 31636 & 53586 & 59198 & 58879 \\
\hline $\mathrm{C}_{4}$ & Sole soybean - Safflower & 116982 & 31636 & 74928 & 87991 & 87887 \\
\hline $\mathrm{C}_{5}$ & Cotton + soybean (4:10)- Chickpea & 121100 & 40006 & 81567 & 81774 & 84327 \\
\hline $\mathrm{C}_{6}$ & Cotton + soybean(4:10)-Linseed & 105360 & 40759 & 69601 & 75447 & 74198 \\
\hline \multirow[t]{3}{*}{$\mathrm{C}_{7}$} & Cotton + soybean $(4: 10)$-Safflower & 120853 & 40577 & 82661 & 94825 & 91147 \\
\hline & S.Em. \pm & 2358 & 474 & 2218 & 1721 & 1214 \\
\hline & C.D.at $5 \%$ & 7266 & 1460 & 6871 & 5303 & 3741 \\
\hline B. & \multicolumn{6}{|l|}{ Sub Plot -Nutrient Management } \\
\hline $\mathrm{F}_{1}$ & $100 \%$ RDF & 108519 & 38054 & 74387 & 78749 & 79023 \\
\hline \multirow[t]{3}{*}{$\mathrm{F}_{2^{-}}$} & $\begin{array}{l}50 \% \mathrm{~N}+100 \% \mathrm{P} \text { and } \mathrm{K} \text { applied through } \\
\text { inorganic fertilizer }+50 \% \mathrm{~N} \text { through Gliricidia }\end{array}$ & 101448 & 34753 & 68914 & 77015 & 74821 \\
\hline & S.Em. \pm & 1212 & 162 & 1341 & 1341 & 737 \\
\hline & C.D.at $5 \%$ & 3678 & 499 & 4067 & NS & 2272 \\
\hline \multirow[t]{3}{*}{ C. } & Interaction effect & & & & & \\
\hline & S.Em. \pm & 32.1 & 1134 & 3547 & 3549 & 1951 \\
\hline & C.D.at $5 \%$ & $\mathrm{NS}$ & NS & NS & NS & NS \\
\hline
\end{tabular}

\begin{tabular}{|c|c|c|c|c|c|c|c|c|}
\hline \multicolumn{9}{|c|}{ Market Rates $\left(\mathrm{Rs}^{-1}\right)$} \\
\hline \multirow{2}{*}{ Crops } & \multicolumn{2}{|c|}{ 2013-14 } & \multicolumn{2}{|c|}{$2014-15$} & \multicolumn{2}{|c|}{$2015-16$} & \multicolumn{2}{|c|}{$2016-17$} \\
\hline & Seed & Stalk & Seed & Stalk & Seed & Stalk & Seed & Stalk \\
\hline Cotton & 5200 & 100 & 5200 & 100 & 4700 & 125 & 4150 & 125 \\
\hline Soybean & 3200 & 75 & 3200 & 75 & 3550 & 100 & 2750 & 125 \\
\hline Chickpea & 2800 & 100 & 2800 & 100 & 3200 & 125 & 3200 & 125 \\
\hline Linseed & 2800 & 100 & 2800 & 100 & 4200 & 100 & 4200 & 125 \\
\hline Safflower & 4500 & 100 & 4500 & 100 & 3200 & 100 & 3200 & 100 \\
\hline
\end{tabular}


Table.6 Net monetary returns as influenced by cropping pattern and nutrient management

\begin{tabular}{|c|c|c|c|c|c|c|}
\hline & \multirow{2}{*}{ Treatments } & \multicolumn{5}{|c|}{ Net monetary returns $\left(\right.$ Rs ha $\left.^{-1}\right)$} \\
\hline & & 2013-14 & 2014-15 & 2015-16 & 2016-17 & Pooled \\
\hline A. & \multicolumn{6}{|c|}{ Main Plot (Cropping Pattern) } \\
\hline $\mathrm{C}_{1}$ & Sole cotton & 55230 & 6793 & 31561 & 37243 & 27919 \\
\hline $\mathrm{C}_{2}$ & Sole soybean-Chickpea & 61614 & 2093 & 21265 & 10813 & 18351 \\
\hline $\mathrm{C}_{3}$ & Sole soybean -Llinseed & 50461 & 2093 & 5823 & 10721 & 10815 \\
\hline $\mathrm{C}_{4}$ & Sole soybean - Safflower & 76973 & 2093 & 27987 & 38997 & 39308 \\
\hline $\mathrm{C}_{5}$ & Cotton + soybean (4:10)- Chickpea & 81267 & 9648 & 37587 & 33864 & 37370 \\
\hline $\mathrm{C}_{6}$ & Cotton + soybean(4:10)-Linseed & 73524 & 9837 & 29058 & 31816 & 30728 \\
\hline \multirow[t]{3}{*}{$\mathrm{C}_{7}$} & Cotton + soybean (4:10)-Safflower & 84574 & 10020 & 41752 & 48929 & 45850 \\
\hline & S.Em. \pm & 2358 & 474 & 2218 & 1721 & 1214 \\
\hline & C.D.at $5 \%$ & 7266 & 1460 & 6871 & 5303 & 3741 \\
\hline B. & \multicolumn{6}{|l|}{ Sub Plot -Nutrient Management } \\
\hline$F_{1}$ & $100 \%$ RDF & 73009 & 7779 & 31954 & 32702 & 33394 \\
\hline \multirow[t]{3}{*}{$\mathrm{F}_{2}$} & $\begin{array}{l}50 \% \mathrm{~N}+100 \% \mathrm{P} \text { and } \mathrm{K} \text { applied through inorganic } \\
\text { fertilizer }+50 \% \mathrm{~N} \text { through Gliricidia }\end{array}$ & 65174 & 4386 & 23769 & 27979 & 26703 \\
\hline & S.Em. \pm & 1212 & 162 & 1341 & 1341 & 737 \\
\hline & C.D.at $5 \%$ & 3678 & 499 & 4067 & 4305 & 2272 \\
\hline \multirow[t]{3}{*}{ C. } & Interaction effect & & & & & \\
\hline & S.Em. \pm & 3211 & 1134 & 3547 & 3549 & 1951 \\
\hline & C.D.at $5 \%$ & NS & NS & NS & NS & NS \\
\hline
\end{tabular}

Table.7 B:C ratio as influenced by cropping pattern and nutrient management

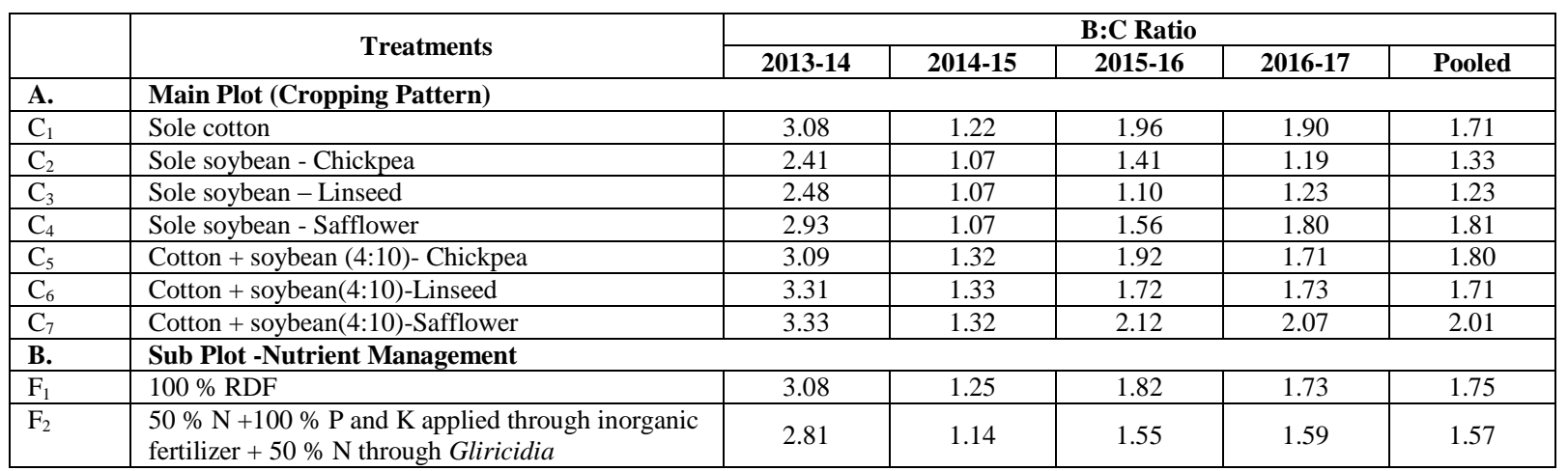

Table.8 Rain water use efficiency as influenced by cropping pattern and nutrient management

\begin{tabular}{|c|c|c|c|c|c|c|}
\hline & \multirow{2}{*}{ Treatments } & \multicolumn{5}{|c|}{ RWUE $\left(\mathrm{kg} \mathrm{ha}^{-1} \mathrm{~mm}^{-1}\right)$} \\
\hline & & 2013-14 & 2014-15 & 2015-16 & 2016-17 & Pooled \\
\hline A. & \multicolumn{6}{|l|}{ Main Plot (Cropping Pattern) } \\
\hline $\mathrm{C}_{1}$ & Sole cotton & 1.62 & 1.74 & 2.32 & 2.53 & 2.14 \\
\hline $\mathrm{C}_{2}$ & Sole soybean - Chickpea & 2.43 & 1.41 & 2.60 & 2.15 & 2.38 \\
\hline $\mathrm{C}_{3}$ & Sole soybean - Linseed & 1.96 & 1.41 & 1.88 & 1.88 & 1.88 \\
\hline $\mathrm{C}_{4}$ & Sole soybean - Safflower & 2.72 & 1.41 & 2.64 & 2.81 & 2.82 \\
\hline $\mathrm{C}_{5}$ & Cotton + soybean (4:10)- Chickpea & 2.73 & 1.77 & 2.89 & 2.60 & 2.68 \\
\hline $\mathrm{C}_{6}$ & Cotton + soybean(4:10)-Linseed & 2.39 & 1.81 & 2.46 & 2.41 & 2.36 \\
\hline \multirow[t]{3}{*}{$\mathrm{C}_{7}$} & Cotton + soybean (4:10)-Safflower & 2.75 & 1.81 & 2.93 & 3.03 & 2.91 \\
\hline & S.Em. \pm & 0.11 & - & 0.08 & 0.06 & 0.04 \\
\hline & C.D.at $5 \%$ & 0.35 & - & 0.24 & 0.17 & 0.12 \\
\hline B. & \multicolumn{6}{|l|}{ Sub Plot -Nutrient Management } \\
\hline$F_{1}$ & $100 \%$ RDF & 2.48 & 1.69 & 2.63 & 2.52 & 2.52 \\
\hline \multirow[t]{3}{*}{$\mathrm{F}_{2}$} & $\begin{array}{l}50 \% \mathrm{~N}+100 \% \mathrm{P} \text { and } \mathrm{K} \text { applied through inorganic } \\
\text { fertilizer }+50 \% \mathrm{~N} \text { through Gliricidia }\end{array}$ & 2.26 & 1.55 & 2.44 & 2.45 & 2.39 \\
\hline & S.Em. \pm & 0.08 & - & 0.05 & 0.04 & 0.02 \\
\hline & C.D.at $5 \%$ & NS & - & 0.14 & NS & 0.07 \\
\hline \multirow[t]{3}{*}{ C. } & Interaction effect & & & & & \\
\hline & S.Em. \pm & 0.20 & - & 0.13 & 0.11 & 0.06 \\
\hline & C.D.at $5 \%$ & NS & - & NS & NS & NS \\
\hline
\end{tabular}


Table.9 Sustainability yield index and value index as influenced by cropping pattern and Nutrient management

\begin{tabular}{|l|l|c|c|}
\hline \multicolumn{1}{|c|}{ Treatments } & $\begin{array}{c}\text { Sustainable } \\
\text { Yield Index } \\
\text { (SYI) }\end{array}$ & $\begin{array}{c}\text { Sustainable } \\
\text { Value Index } \\
\text { (SVI) }\end{array}$ \\
\hline A. & Main Plot (Cropping Pattern) & & \\
\hline $\mathrm{C}_{1}$ & Sole cotton & 0.65 & 0.45 \\
\hline $\mathrm{C}_{2}$ & Sole soybean - Chickpea & 0.77 & 0.34 \\
\hline $\mathrm{C}_{3}$ & Sole soybean - Linseed & 0.61 & 0.19 \\
\hline $\mathrm{C}_{4}$ & Sole soybean - Safflower & 0.90 & 0.72 \\
\hline $\mathrm{C}_{5}$ & Cotton + soybean (4:10)- Chickpea & 0.87 & 0.71 \\
\hline $\mathrm{C}_{6}$ & Cotton + soybean(4:10)-Linseed & 0.76 & 0.57 \\
\hline $\mathrm{C}_{7}$ & Cotton + soybean(4:10)-Safflower & 0.94 & 0.85 \\
\hline B. & Sub Plot -Nutrient Management & & 0.61 \\
\hline $\mathrm{F}_{1}$ & 100\% RDF & 0.81 & 0.48 \\
\hline $\mathrm{F}_{2}$ & $\begin{array}{l}\text { 50\% N +100\% P and K applied through } \\
\text { inorganic fertilizer + 50 \% N through }\end{array}$ & 0.76 & \\
\hline
\end{tabular}

Table.10 System productivity and system profitability of various cropping systems as influenced by cropping pattern and nutrient management

\begin{tabular}{|c|c|c|c|c|c|c|}
\hline & \multirow[t]{2}{*}{ Treatments } & \multirow{2}{*}{$\begin{array}{c}\text { Total } \\
\text { crop } \\
\text { duration } \\
\text { of } \\
\text { sequence } \\
\text { (days) }\end{array}$} & \multicolumn{2}{|c|}{$\begin{array}{c}\text { System Productivity } \\
\left(\mathrm{kg} \mathrm{ha}^{-1} \mathrm{day}^{-1}\right)\end{array}$} & \multicolumn{2}{|c|}{$\begin{array}{l}\text { System Profitability } \\
\left(\text { Rs ha }^{-1} \text { day }^{-1}\right)\end{array}$} \\
\hline & & & \begin{tabular}{|c|} 
Crop \\
Productivity
\end{tabular} & $\begin{array}{l}\text { System } \\
\text { Producti } \\
\text { vity }\end{array}$ & $\begin{array}{c}\text { Crop } \\
\text { Profitability }\end{array}$ & $\begin{array}{c}\text { System } \\
\text { Profitability }\end{array}$ \\
\hline A. & \multicolumn{6}{|l|}{ Main Plot (Cropping Pattern) } \\
\hline $\mathrm{C}_{1}$ & Sole cotton & 214 & 7.25 & 4.25 & 130.46 & 76.49 \\
\hline $\mathrm{C}_{2}$ & Sole soybean - Chickpea & 245 & 7.04 & 4.73 & 74.90 & 50.28 \\
\hline $\mathrm{C}_{3^{-}}$ & Sole soybean - Linseed & 253 & 5.38 & 3.73 & 42.75 & 29.63 \\
\hline $\mathrm{C}_{4^{-}}$ & Sole soybean - Safflower & 255 & 8.01 & 5.59 & 154.15 & 107.69 \\
\hline $\mathrm{C}_{5}$ & Cotton +soybean (4:10)- Chickpea & 245 & 7.93 & 5.32 & 152.53 & 102.38 \\
\hline $\mathrm{C}_{6}$ & Cotton +soybean (4:10)-Linseed & 253 & 6.75 & 4.68 & 121.45 & 84.19 \\
\hline $\mathrm{C}_{7}$ & Cotton+ soybean (4:10)- Safflower & 255 & 8.25 & 5.76 & 179.80 & 125.62 \\
\hline & S.Em. \pm & & 0.13 & 0.08 & 5.47 & 3.33 \\
\hline & C.D.at $5 \%$ & & 0.39 & 0.24 & 16.8 & 10.3 \\
\hline B. & \multicolumn{6}{|l|}{ Sub Plot -Nutrient Management } \\
\hline $\mathrm{F}_{1}$ & $100 \% \mathrm{RDF}$ & & 7.43 & 5.00 & 136.28 & 91.49 \\
\hline $\mathrm{F}_{2}$ & $\begin{array}{l}50 \% \mathrm{~N}+100 \% \mathrm{P} \text { and K applied } \\
\text { through inorganic fertilizer }+50 \% \mathrm{~N} \\
\text { through Gliricidia }\end{array}$ & & 7.02 & 4.73 & 108.31 & 73.16 \\
\hline & S.Em. \pm & & 0.07 & 0.05 & 3.24 & 2.02 \\
\hline & C.D.at $5 \%$ & & 0.23 & 0.14 & 9.99 & 6.23 \\
\hline C. & Interaction effect & & & & & \\
\hline & S.Em. \pm & & 0.20 & 0.12 & 8.58 & 5.35 \\
\hline & C.D.at $5 \%$ & & NS & NS & NS & NS \\
\hline
\end{tabular}


Table.11 Ancillary observation of cotton as influenced by cropping pattern and nutrient management (2013-14 to 2016-17)

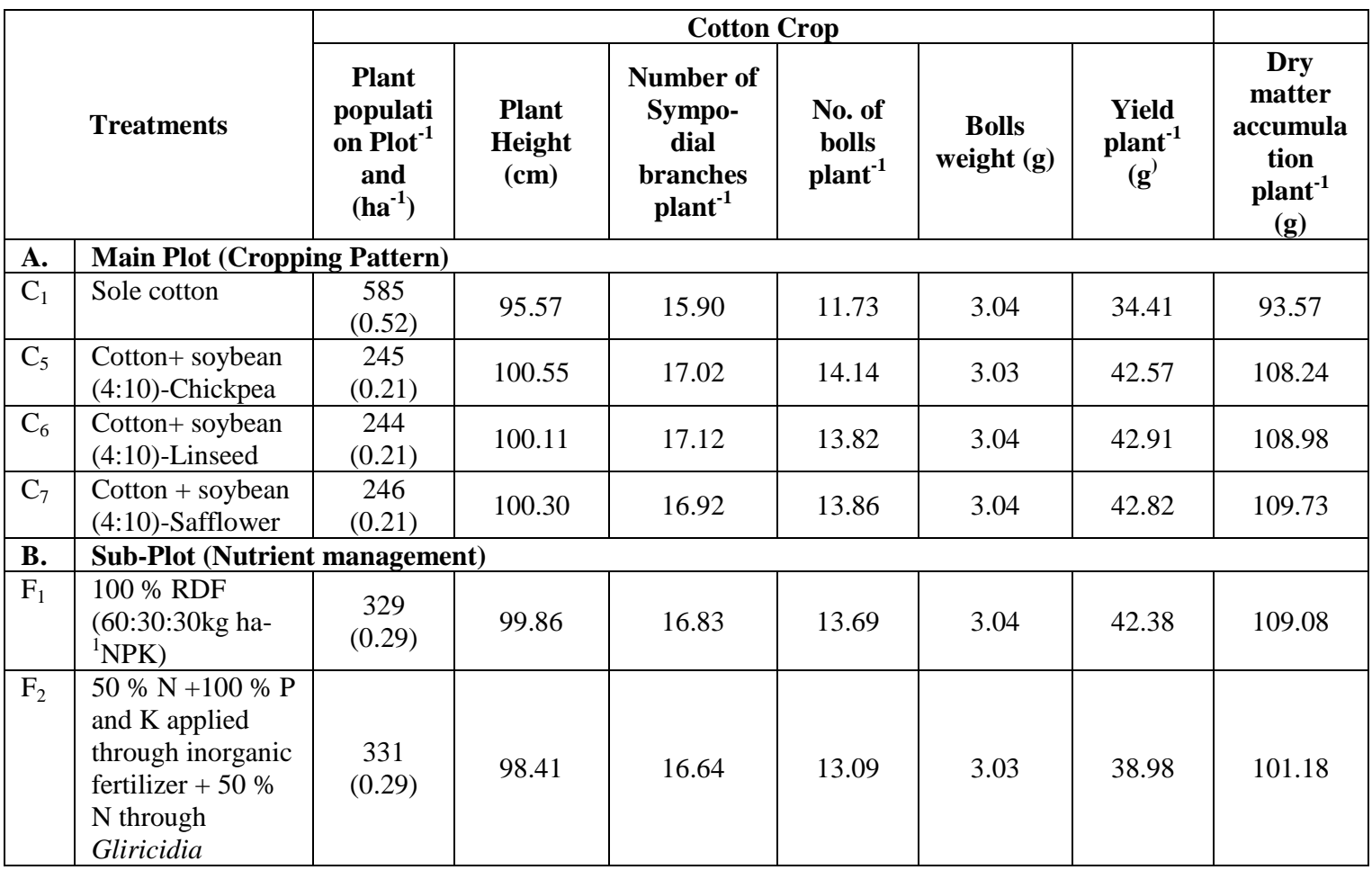

Table.12 Ancillary observation of soybean as influenced by cropping pattern and nutrient management (2013-14 to 2016-17)

\begin{tabular}{|c|c|c|c|c|c|c|}
\hline \multirow{2}{*}{\multicolumn{2}{|c|}{ Treatments }} & \multicolumn{5}{|c|}{ Soybean Crop } \\
\hline & & \multirow{2}{*}{$\begin{array}{l}\text { Plant } \\
\text { population } \\
\text { Plot }^{-1}\left(\text { ha }^{-1}\right)\end{array}$} & \multirow{2}{*}{$\begin{array}{l}\text { Plant } \\
\text { Height } \\
\text { (cm) }\end{array}$} & \multirow{2}{*}{$\begin{array}{l}\text { Number of } \\
\text { bran-ches } \\
\text { plant }^{-1}\end{array}$} & \multirow{2}{*}{$\begin{array}{l}\text { Num- } \\
\text { ber of } \\
\text { pods } \\
\text { plant }^{-1}\end{array}$} & \multirow{2}{*}{$\begin{array}{l}\text { Yield } \\
\text { plant }^{-1} \\
\left(g^{)}\right.\end{array}$} \\
\hline A. & Main Plot (Cropping Pattern) & & & & & \\
\hline $\mathrm{C}_{1}$ & Cotton sole & - & - & - & - & - \\
\hline $\mathrm{C}_{2}$ & Soybean sole - Chickpea & $\begin{array}{c}1257 \\
(3.98) \\
\end{array}$ & 55.71 & 4.91 & 33.29 & 5.15 \\
\hline $\mathrm{C}_{3}$ & Soybean sole - Linseed & $\begin{array}{c}1257 \\
(3.98) \\
\end{array}$ & 55.71 & 4.91 & 33.29 & 5.15 \\
\hline $\mathrm{C}_{4}$ & Soybean sole - Safflower & $\begin{array}{c}1257 \\
(3.98) \\
\end{array}$ & 55.71 & 4.91 & 33.29 & 5.15 \\
\hline $\mathrm{C}_{5}$ & Cotton + soybean (4:10)-Chickpea & $\begin{array}{c}679 \\
(2.15) \\
\end{array}$ & 55.68 & 4.99 & 32.07 & 4.95 \\
\hline $\mathrm{C}_{6}$ & Cotton + soybean (4:10)-Linseed & $\begin{array}{c}672 \\
(2.12) \\
\end{array}$ & 55.43 & 5.01 & 32.45 & 4.84 \\
\hline $\mathrm{C}_{7}$ & Cotton + soybean (4:10)-Safflower & $\begin{array}{c}681 \\
(2.15) \\
\end{array}$ & 55.47 & 4.86 & 33.57 & 4.98 \\
\hline B. & \multicolumn{6}{|l|}{ Sub-Plot (Nutrient Management) } \\
\hline $\mathrm{F}_{1}$ & $100 \%$ RDF (60:30:30kg ha- $\left.{ }^{1} \mathrm{NPK}\right)$ & $\begin{array}{c}970 \\
(3.07)\end{array}$ & 55.72 & 5.02 & 33.45 & 5.08 \\
\hline $\mathrm{F}_{2}$ & $\begin{array}{l}50 \% \mathrm{~N}+100 \% \mathrm{P} \text { and } \mathrm{K} \text { applied } \\
\text { through inorganic fertilizer }+50 \% \mathrm{~N} \\
\text { through Gliricidia }\end{array}$ & $\begin{array}{c}965 \\
(3.05)\end{array}$ & 55.52 & 4.85 & 32.54 & 4.98 \\
\hline
\end{tabular}


Table.13 Ancillary observation of chickpea, linseed and safflower crops as influenced by cropping pattern and nutrient management (Average of three years 2013-14 to 2016-17)

\begin{tabular}{|c|c|c|c|c|c|c|}
\hline Tre & ments & $\begin{array}{c}\text { Plant } \\
\text { population } \\
\text { Plot }^{-1}\left(\mathrm{ha}^{-1}\right)\end{array}$ & $\begin{array}{l}\text { Plant Height } \\
\text { (cm) }\end{array}$ & $\begin{array}{c}\text { Number of } \\
\text { branches } \\
\text { plant }^{-1}\end{array}$ & $\begin{array}{c}\text { Number of } \\
\text { pods/bolls/ros } \\
\text { sette plant }^{-1}\end{array}$ & $\begin{array}{c}\text { Yield } \\
\text { plant }^{-1} \\
\left(g^{\prime}\right)\end{array}$ \\
\hline A. & $\begin{array}{l}\text { Cropping } \\
\text { Pattern }\end{array}$ & \multicolumn{5}{|c|}{ Chickpea } \\
\hline $\mathrm{C}_{2}$ & $\begin{array}{l}\text { Sole soybean- } \\
\text { Chickpea }\end{array}$ & $\begin{array}{l}1017 \\
(3.31)\end{array}$ & 54.72 & 4.88 & 27.21 & 3.66 \\
\hline $\mathrm{C}_{5}$ & $\begin{array}{l}\text { Cotton + soybean } \\
(4: 10) \text {-Chickpea }\end{array}$ & $579(1.83)$ & 57.27 & 4.95 & 26.23 & 3.70 \\
\hline B. & \multicolumn{2}{|c|}{ Nutrient Management } & & & & \\
\hline $\mathrm{F}_{1}$ & $\begin{array}{ll}100 \% & \text { RDF } \\
(60: 30: 30 \mathrm{~kg} & \text { ha- }^{-} \\
\text {NPK) } & \end{array}$ & $\begin{array}{l}815 \\
(2.57)\end{array}$ & 54.68 & 4.94 & 26.58 & 3.70 \\
\hline $\mathrm{F}_{2}$ & $\begin{array}{l}50 \% \mathrm{~N}+100 \% \\
\mathrm{P} \text { and } \mathrm{K} \text { applied } \\
\text { through inorganic } \\
\text { fertilizer }+50 \% \\
\mathrm{~N} \text { through } \\
\text { Gliricidia }\end{array}$ & $810(2.56)$ & 54.31 & 4.90 & 26.86 & 3.66 \\
\hline A. & $\begin{array}{l}\text { Cropping } \\
\text { Pattern } \\
\end{array}$ & \multicolumn{5}{|c|}{ Linseed } \\
\hline $\mathrm{C}_{3}$ & $\begin{array}{l}\text { Sole soybean- } \\
\text { Linseed }\end{array}$ & $322(1.02)$ & 40.95 & 5.17 & 28.16 & 2.86 \\
\hline $\mathrm{C}_{6}$ & $\begin{array}{l}\text { Cotton + soybean } \\
(4: 10) \text {-Linseed }\end{array}$ & $101(0.35)$ & 43.23 & 5.07 & 28.77 & 2.87 \\
\hline B. & \multicolumn{2}{|c|}{ Nutrient Management } & & & & \\
\hline$\overline{F_{1}}$ & $\begin{array}{ll}100 \% & \text { RDF } \\
(60: 30: 30 \mathrm{~kg} & \text { ha- }^{-} \\
\text {NPK) } & \end{array}$ & $216(0.68)$ & 42.09 & 5.12 & 28.84 & 2.84 \\
\hline $\mathrm{F}_{2}$ & $\begin{array}{l}50 \% \mathrm{~N}+100 \% \\
\mathrm{P} \text { and } \mathrm{K} \text { applied } \\
\text { through inorganic } \\
\text { fertilizer }+50 \% \\
\mathrm{~N} \text { through } \\
\text { Gliricidia }\end{array}$ & $217(0.68)$ & 42.09 & 5.13 & 28.08 & 2.89 \\
\hline A. & $\begin{array}{l}\text { Cropping } \\
\text { Pattern }\end{array}$ & \multicolumn{5}{|c|}{ Safflower } \\
\hline $\mathrm{C}_{4}$ & $\begin{array}{l}\text { Sole soybean- } \\
\text { Safflower }\end{array}$ & $309(0.97)$ & 80.10 & 6.81 & 13.89 & 12.09 \\
\hline $\mathrm{C}_{7}$ & $\begin{array}{l}\text { Cotton + soybean } \\
(4: 10) \text {-Safflower }\end{array}$ & $168(0.53)$ & 81.75 & 6.67 & 14.04 & 12.06 \\
\hline B. & \multicolumn{2}{|c|}{ Nutrient Management } & & & & \\
\hline $\mathrm{F}_{1}$ & $\begin{array}{ll}100 \% & \text { RDF } \\
(60: 30: 30 \mathrm{~kg} & \text { ha- }^{-} \\
\text {NPK) } & \\
\end{array}$ & $238(0.75)$ & 81.44 & 6.83 & 14.22 & 12.06 \\
\hline $\mathrm{F}_{2}$ & $\begin{array}{l}50 \% \mathrm{~N}+100 \% \\
\mathrm{P} \text { and } \mathrm{K} \text { applied } \\
\text { through inorganic } \\
\text { fertilizer }+50 \% \\
\mathrm{~N} \text { through } \\
\text { Gliricidia }\end{array}$ & $239(0.75)$ & 80.42 & 6.85 & 13.71 & 12.09 \\
\hline
\end{tabular}


Table.14 pH, EC, OC, available nitrogen, phosphorus and potassium as influenced by cropping pattern and nutrient management

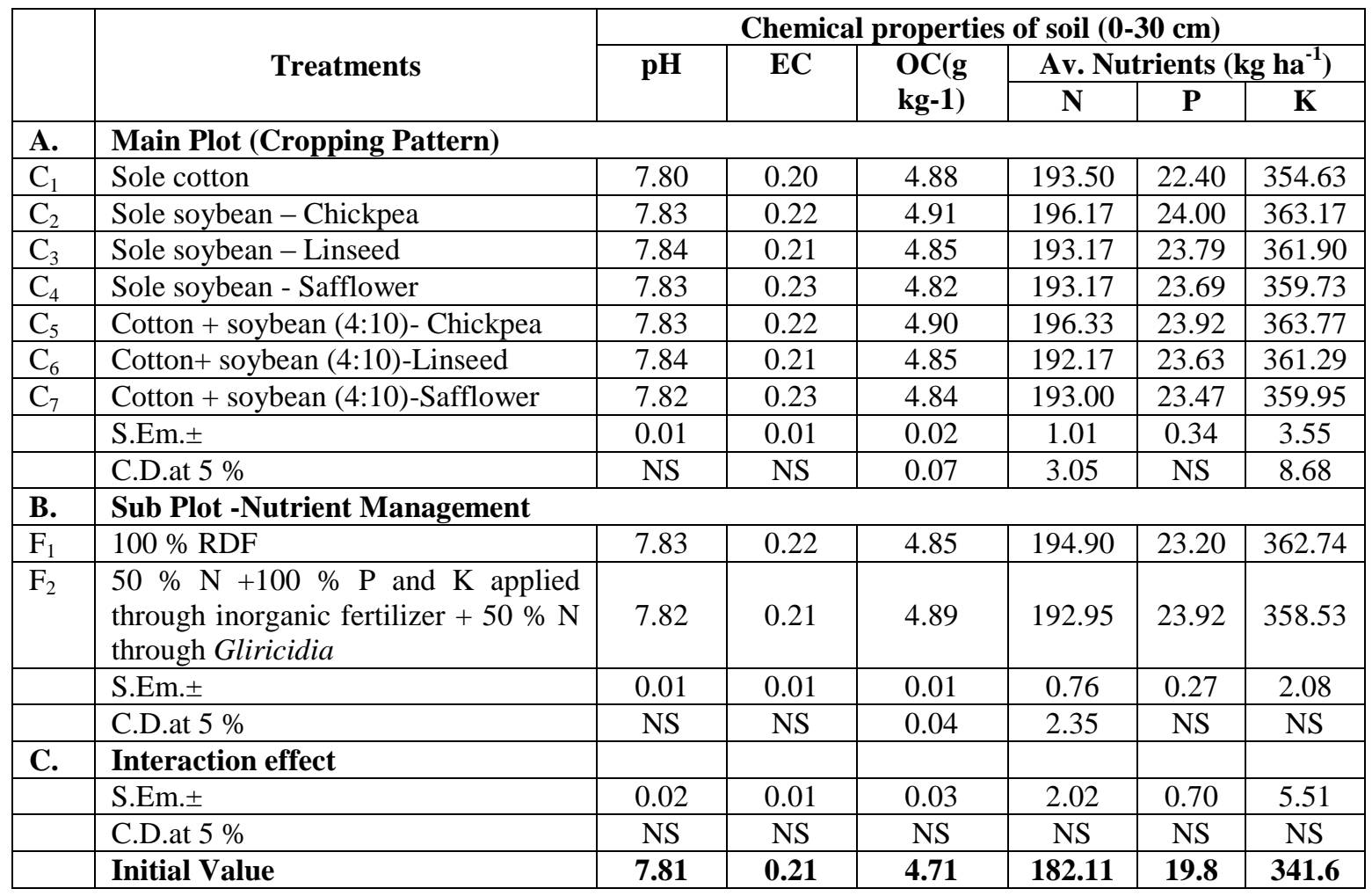

It might be due to higher seed cotton equivalent yield obtained under RDF. Behera et al., (2002) reported that fertilizing the cotton recorded significantly higher water use efficiency than lower levels of nitrogen, 120 and $80 \mathrm{~kg} / \mathrm{ha}$. Reduction in nitrogen dose tended to decrease water use efficiency; it was partially because of marked decline in transpiring surface, less stomatal conductance and less extraction of available soil moisture in plant.

Sustainable yield index and sustainable value index

Sustainable yield index and value index recorded higher value in the treatments of Cotton + soybean (4:10)-safflower (0.94 and 0.85) followed by the treatment of sole soybean - sole safflower cropping system (0.90 and 0.72). In nutrient management treatment of $100 \%$ RDF recorded the higher sustainable yield index and sustainable value index (0.81 and 0.61) (Table 9).

Wanjari et al., (2004) reported that maximum SYI $037-0.62$ achieved for $100 \%$ NPK + FYM treatment in almost all the locations indicating the beneficial effect of integrated use of nutrients on sustaining higher crop productivity by correcting marginal deficiencies of secondary and micronutrients. Judicious application of inorganic and organic nutrients in an integrated manner is essential for proper nutrient supply, sustaining crop productivity and soil quality in long term cropping system. Katkar et al., (2012) reported that the in long term yield data, both the crops was used to study sustainability yield index which were found to be relatively higher in sorghum - wheat cropping sequence with super optimal fertilizer dose (0.436) followed by $100 \%$ RDF + FYM (0.432) and $100 \% \mathrm{RDF}+$ sulphur (0.421). Same findings 
also reported by Srinivasa rao et al., (2012),combined use of organic manure (crop residues and FYM) along with chemical fertilizer enhancing the sustainability sequestration in a soybean-safflower sequence cropping system under rainfed conditions on Vertisols in central India.

\section{Productivity and profitability of cropping systems}

Crop productivity of cropping system on actual cropping duration on the basis of cotton equivalent yield was noticed significantly higher in the treatment of Cotton + soybean (4:10)-safflower cropping system $\left(8.25 \mathrm{~kg} \mathrm{ha}^{-1}\right.$ day $^{-1}$ ) and found being at par with the treatment of sole soybean - safflower and Cotton + soybean $(4: 10)$ - chickpea cropping system i.e. 8.01 and $7.93 \mathrm{~kg} \mathrm{ha}^{-1}$ day $^{-1}$, respectively (Table 10).

However, system productivity was recorded significantly higher value in the treatment of Cotton + soybean (4:10)- safflower and found being at par with the treatment sole soybeansafflower cropping system i.e. 5.76 and $5.59 \mathrm{~kg} \mathrm{ha}^{-1}$ day $^{-1}$, respectively.

Crop profitability and system profitability of cropping system on the basis of net monetary returns was noticed significantly higher in the treatment of Cotton + soybean (4:10)safflower (179.80 and $125.62 \mathrm{Rs} \mathrm{ha}^{-1} \mathrm{day}^{-1}$ ) than rest of the treatments (Table 6).

In nutrient management, treatment of $100 \%$ RDF recorded the higher system productivity and system profitability i.e. 7.43 and $5.00 \mathrm{~kg}$ $\mathrm{ha}^{-1}$ day $^{-1}$ and 136.28 and 91.49 $\mathrm{Rs} \mathrm{ha}^{-1} \mathrm{day}^{-1}$, respectively.

Crop duration was numerically found maximum (245, 253 and 255 days) under treatments of sole soybean chickpea/linseed/safflower and Cotton + soybean (4:10)-chickpea / linseed /safflower than the treatment of sole cotton.

A cropping systems approach may offer opportunities for producers to increase economic returns. Management of dynamic cropping systems will need to be based not only on single-year profit opportunities, but also on subsequent crop sequence effects. Thus, to more productivity and market rates of the mixed crops and low cost of cultivation also helped in increasing higher cotton equivalent yield and economics returns which ultimately effects on system productivity and profitability. Cotton + sorghum-ragi, followed by cotton-sunflower -ragi and cotton-maize ragi sequences were more profitable and economically viable than sole cotton (Jagvirsingh et al., 2000).Soybean-mustardgroundnut, soybean-coriander-wheat, soybean-Isabgol-groundnut recorded higher NMR, B:C ratio and system productivity in Western Vidarbha zone of Maharashtra (Anonymous, 2008).

\section{Growth and yield attributing characters}

Average of three years showed that, plant height, number of sympodial branches, number of bolls, yield and dry matter accumulation per plant recorded higher value in the treatments of Cotton + soybean (4:10) chickpea /linseed/ safflower cropping patterns as compared to the treatment of sole cotton. However, boll weight was recorded similar value in the treatments of sole cotton as well as cotton + soybean $(4: 10)$ row proportions followed by the rabi crops chickpea / linseed and safflower.

However, in respect of ancillary observation in soybean, chickpea, linseed and safflower crops did not influenced by the treatments of sole cotton, sole soybean (4:10) - chickpea, linseed and safflower cropping system and registered the more or less equal in plant 
height, number of branches, number of pods/balls/rossets plant $^{-1}$ in chickpea, linseed and safflower plant $^{-1}$ in respective crops (Tables 11-13).

In respect of nutrient management treatments, plant height, number of branches, number of bolls, yield and dry matter accumulation per plant recorded higher value in the treatment of $100 \mathrm{RDF}$ than $50 \% \mathrm{~N}+100 \% \mathrm{P}$ and $\mathrm{K}$ applied through inorganic fertilizer $+50 \% \mathrm{~N}$ through Gliricidia. Same trend of results are also recorded in the treatments of ancillary observation of the soybean, chickpea linseed and safflower crops. However, cropping system and nutrient management did not influence the bolls weight.

\section{Chemical properties}

The data in respect of $\mathrm{pH}$ and $\mathrm{EC}$ was found to be non-significant due to different cropping system as well as by the application of nutrient management treatments. One of the most important chemical properties of soil as a medium for plant growth is its $\mathrm{pH}$. Effect of anion - cationim balance in crop plants affects soil $\mathrm{pH}$.

Lad and Patel (1965) found no change in soil $\mathrm{pH}$ by growing the same crop as well as by rotational system of cropping. Timsina et al., (2001) reported a slight decrease in $\mathrm{pH}$ after three years of rice-wheat cropping system in Bangladesh. The above literature indicates differential response of cropping systems on soil $\mathrm{pH}$. Soil environment and type of crops grown under different cropping systems play a significant role in relation to soil $\mathrm{pH}$. Gawai (2003) reported reduction in soil $\mathrm{pH}$, only due to microbial decomposition of organic manures (Table 14).

In different cropping patterns, the maximum organic carbon content in soil was recorded in the treatments of sole soybean - chickpea and being at par with rest of the treatments and significantly superior than sole soybean safflower treatments. Among sub plot treatments application of different levels of fertilizers i.e. $100 \% \mathrm{RDF}$ and $50 \% \mathrm{~N}+100 \%$ $\mathrm{P}$ and $\mathrm{K}$ applied through inorganic fertilizer + $50 \% \mathrm{~N}$ through Gliricidia was found being at par (4.89 and $4.85 \mathrm{~kg} \mathrm{ha}^{-1}$ ).

Different cropping systems have different levels of uptake of plant nutrients. However, uptake of nutrients by same or similar cropping system over the years on the same land results in mining of similar nutrients from the soil affecting its fertility status. This might be due to buildup of higher amount of organic carbon in the soil after harvest of the crop which is due to addition of higher biomass to soil. Legume crop like chickpea add crop biomass in soil. Organic carbon content of soil was improved in all treatments except control. Application of organic fertilizer and/ or combination with chemical fertilizer helped for building up of organic carbon. Sharma and Subehia (2003) also reported greater levels of soil organic carbon under integrated treatments of organic and inorganic combinations. Addition of fertilizer and integrated nutrients management generally results in buildup of available nutrients, more so under rainfed conditions. In our present investigation it was found that organic carbon increased with addition of 50 $\% \mathrm{~N}$ through gliricidia leaves. This is because of fewer uptakes of nutrients by cotton and soybean crops.

Available $\mathrm{N}$ status indicates that the $\mathrm{N}$ status of the soil was found to be high as compared to initial value $\left(182.11 \mathrm{~kg} \mathrm{ha}^{-1}\right)$. Maximum available $\mathrm{N}$ obtained was 196.33 and 196.17 $\mathrm{kg} \mathrm{ha}{ }^{-1}$ recorded in the treatment of sole soybean-chickpea and Cotton + soybean (4:10)-chickpea and being at par with the rest of the treatments. Treatments of soybean + chickpea and cotton +soybean (4:10)- 
chickpea recorded significantly higher available $\mathrm{N}$ than by sole cotton treatments, it means there was more mining of nutrients from directly available nutrient from fertilizer. Similar results have also been reported by Singh et al., (2001).

An application of treatment $100 \%$ RDF which was significantly higher over other treatment. The lowest value of available $\mathrm{N}$ (192.95 $\mathrm{kg} \mathrm{ha}^{-1}$ ) was observed in the INM plot. Hence, it is clear that application of chemical fertilizers increased the available $\mathrm{N}$ of soil, which may be attributed to mineralization of $\mathrm{N}$.

Available $\mathrm{P}$ and $\mathrm{K}$ status of the surface soil did not shown the significant differences in cropping patterns and also in nutrients management treatments. The value of available phosphorus and potassium increased than initial value with application of RDF as well as by INM. Vertisols are generally rich in $\mathrm{K}$ content, and application of potassic fertilizers is not recommended in some pockets to these soils. Nevertheless, the importance of $\mathrm{K}$ in regulating and improving water functions in plant system and enabling the crop to withstand drought under rainfed conditions where intermittent dry spells are usual, cannot be undermined. Similar, observation was made by Bharadwaj et al., (1994).

Hence, it can be recommended that, among the various treatments combination cotton + soybean (4:10)-safflower cropping sequence sown with tractor drawn seed drill with application of 100\% RDF (45:55:30 kg ha ${ }^{1} \mathrm{NPK}$ ) (through inorganic fertilizer) in kharif season and 22:13.75:00 kg ha ${ }^{-1} \mathrm{NPK}$ (through inorganic fertilizer)to safflower in rabi season recorded significantly higher cotton equivalent yield, net monetary returns, B:C ratio, RWUE, sustainable value and yield index, system productivity and profitability than rest of the treatments combinations.

\section{Acknowledgement}

The authors are grateful to the All India Coordinated Research Project for Dryland Agriculture, CRIDA (ICAR) Hyderabad, for providing financial support for conducting the research under study.

\section{References}

Anonumous, 2008. Efficient cropping system in Western Vidarbha Zone of Maharashtra.185-191.

Behera, U.K., Ruwali, K.N., Verma, P.K. and Pandey, H.N. 2002. Productivity and water use efficiency of macaroni (Triticum durum) and bread wheat (Triticum aestivum) under varying irrigation levels and schedules in the vertisols of central India. Indian $\mathrm{J}$. Agron. 47 (4), 518-525.

Bharadwaj, R. B. L., and Tandon, H. L. S. 1981. Fertilizer use research in some wheat based cropping system. Fertilizer News. 26(9): 23-32.

Bhartati, V., Nandan, R., Kumar, V., and Pandey I.B.2007. Effect of irrigation levels on yield, water use efficiency and economics of winter maize (Zea mays) - based intercropping systems. Indian Journal of Agronomy. 52 (1): 27-30.

Chand Ramesh, and Chauhan Sonia 2002. Socio-economic factors in agricultural diversification in India. Agricultural situation in India. 2002. pp.523-529.

Chittapur, B.M. 2004. Cotton based cropping systems for sustained productivity. International Symposium on "Strategies for Sustainable Cotton Production: A Global Vision". Crop Production, 23-25 November, University of Agriculture Science, Dharwad, Karnataka (India)

De, Rajat and Singh, S.1981. Management practices for intercropping systems. In International Workshop on Intercropping, ICRISAT, Hyderabad, 
India. Pp. 17-24.

Different levels of fertility and spacing. Indian J. Agron. 45 (4):776-781.

Dutta, D., and Bandyopadhyay, P. 2006. Production potential of groundnut with pigeonpea and maize under various row proportions in rainfed Alfisols of West Bengal. Indian Journal of Agronomy 51(2):103-106.

Gawai, P. P. 2003. Effect of integrated nutrient management system in sorghum-chickpea cropping sequence. Ph.D. thesis submitted to M.P.K.V., Rahuri (M.S.) pp. 80-167.

Gawai, P. P., and Pawar, V.S.2005.Yield and yield components of sorghum (Sorghum bicolor) as influenced by integrated nutrient management system and its residual effect on chickpea (Cicer arietinum). Ann. Agric. Res., 26(3): 378-382.

Gill, M.S., and Ahlawat, I.P.S. 2006. Crop diversification and its role towards sustainability and profitability. Indian Journal of fertilizers.2 (9):125-138, 150.

Goswami, V.K., Kaushik, S.K. and Gautam, R.C. 2002. Effect of intercropping and weed control on nutrient uptake and water use efficiency of pearlmillet (Pennisetum glaucum) under rainfed conditions. Indian Journal of Agronomy 47(4), 504-508.

Gupta, R.P. and Tewari, S.K. 1985. Factors affecting crop diversification: A critical Analysis. Indian Journal of Agricultural economics.40 (3):304-309.

Hulugalle, N.R., and Lal, R. 1986. Soil-water balance of intercropped maize and cowpea grown in a tropical hydromorphic soil in western Nigeria. Agron. J. 77, 86-90.

Jagvirsingh, Venugopal M.V., and Kairon M.S. 2000. Effect of cotton based cropping system and nutrient management in the productivity and soil fertility of rainfed vertisols. Extended summaries. International conference on managing natural resources, February 14-18, New Delhi, India, Vol 3:945946.

Katkar, R.N., V. K. Kharche, B. A. Sonune, R. H. Wanjari And MuneshwarSingu 2012. Long term effect of nutrient management on soil quality and sustainable productivity under sorghumwheat crop sequence in Vertisol of Akola. Agropedology, 22 (2): J03-JJ4

Sankaranarayanan, K., Nalayini P. and Praharaj 2012. Multitier cropping system to enhance resource utilization, profitability and sustainability of Bt. cotton (Gossypium hirsutum) production system. Indian Journal of Agricultural Science 82 (12):10441050.

Sharma, S. P., and Subehia S. K. 2003. Effects of twenty-five years of fertilizer use on maize and wheat yields and quality of an acidic soil in the western Himalayas. Experimental Agric., 39:5563.

Singh, S. K., Verma S. C. and Singh R. P. 2001. Effect of integrated nutrient management on yield, nutrient on productivity, nutrients uptake, water use and profitability of pearlmillet under rainfed condition. Indian Journal of Agronomy, 51 (4):263-266.

Srinivasarao, Ch., Venkateswarlu, B., Lal, R., Singh, A. K., Kundu, S., Vittal, K. P. R., Sharma, S. K., Sharma, R. A., Jain, M. P. and Chary, G. R. 2012. Sustaining agronomic productivity and quality of a Vertisolic Soil (Vertisol) under soybean-safflower cropping system in semi-arid central India. Can. J. Soil Sci. 92: 771-785.

Tanaka, D.L., J.M. Krupinsky, S.D. Merrill, M.A. Liebig, and J.D. Hanson 2007. Dynamic cropping systems

Tetarwal, J.P., and Rana K.S.2006. Impact of cropping system, fertility levels and 
moisture conservation practices the sustainability of long-term intensive cropping systems in India. Journal of Sustainable Agriculture 24 (4):39-56.

Timsina, J., Singh, U., Badaruddin, M., Meisner, C., Amin, M.R.2001. Cultivar, nitrogen, and water effects on productivity, and nitrogen-use efficiency and balance for rice-wheat sequences of Bangladesh. Field Crops Res. 72, 143-161.

Tomar, R.S.S., and Julka R.1997. Effect of fertility levels and spacing on growth and yield of hirsutum cotton

Tomar, R.S.S., Kushwaha A.L., Julka R. and Madloi K.C. 2000. Productivity of upland cotton genotypes under uptake and changes in soil fertility under rice - lentil cropping system. Indian J. of Agron, 36(2): 191-197.

Venugopal, K., Gopalkrishnan $\mathrm{N}$ and Guruanajan K.N.2000.Sustainability of production potential of cotton and cotton based cropping system. Extended summaries. International conference on managing natural resources, February 14-18, New Delhi, India, Vol 3:947949.

Wanjari, R. H., M. V. Singh, and P. K. Ghosh, 2004. Sustainable yield index: An approach to evaluate the sustainability of long-term intensive cropping systems in India. Journal of Sustainable Agriculture 24 (4):39-56.

\section{How to cite this article:}

Turkhede, A.B., M.B. Nagdeve, A.P. Karunakar, V.V. Gabhane, V.D. Mohod and Mali, R.S. 2017. Diversification in Cotton Based Cropping System under Mechanization in Rainfed Condition of Vidarbha of Maharashtra. Int.J.Curr.Microbiol.App.Sci. 6(9): 2189-2206. doi: https://doi.org/10.20546/ijcmas.2017.609.269 